\title{
A Comparison of GPS, VLBI and Model Estimates of Ocean Tide Loading Displacements
}

Ian D. Thomas (corresponding author), Matt A. King, Peter J. Clarke.

School of Civil Engineering and Geosciences, Newcastle University, Newcastle upon Tyne, NE2 1JU, UK

Tel: +44 (0)191222 6323

Fax: +44 (0)1912226502

E-mail: Ian.Thomas@newcastle.ac.uk

\section{Abstract}

In recent years, Ocean Tide Loading Displacements (OTLD) have been measured using the Global Positioning System (GPS) and Very Long Baseline Interferometry (VLBI). This study assesses the accuracy of GPS measurements of OTLD by comparison with VLBI measurements and estimates derived from numerical ocean tide models. A daily precise point positioning (PPP) analysis was carried out on $\sim 11$ years of GPS data for each of 25 sites that have previous OTLD estimates based on data from co-located VLBI sites. Ambiguities were fixed to integer values where possible. The resulting daily estimates of OTLD, at eight principal diurnal and semi-diurnal tidal frequencies, were combined to give GPS measurements of OTLD at each site. The threedimensional GPS and VLBI measurements of OTLD were compared with estimates computed (by convolution with Green's functions) from five modern ocean tide models (CSR4.0, FES2004, GOT00.2,

NAO99b,TPXO6.2). The GPS / model agreement is shown to be similar to the VLBI / model agreement. In the important radial direction, the GPS / model misfit is shown to be smaller than the VLBI / model misfit for seven of the eight tidal constituents; the exception being the K2 constituent. Fixing of GPS carrier phase ambiguities to integer values resulted in marginal improvement to the GPS / model agreement. Statistically, it is shown there is no significance to the difference between the fit of the GPS and VLBI measurements of OTLD to modelled values. Equally, differences in fit of either the complete set of GPS or VLBI estimates to the five sets of model derived values cannot be identified with statistical significance. It is thus concluded that, overall, we cannot distinguish between GPS and VLBI measurements of OTLD, and that at the global scale, present ocean tide models are accurate to within the current measurement noise of these techniques.

Keywords: GPS, Ocean Tide Loading (OTL), Ocean Tide Loading Displacement (OTLD), VLBI, Ocean tide models.

This is the authors' version of a manuscript that has been accepted by J ournal of Geodesy. The final publication is available at Springer via doi:10.1007/s00190-006-0118-9 


\subsection{Introduction}

Since the late 1980s, geodetic techniques capable of measuring harmonic site displacements with millimetre-level accuracies (Allinson et al. 2004; Schenewerk et al. 2001), and corresponding gravitational variations with sub-0.1 $\mu \mathrm{Gal}$ accuracies (Baker and Bos 2003) have been demonstrated at diurnal and semi-diurnal tidal frequencies.

VLBI has been shown to be capable of determining both radial (Sovers 1994) and threedimensional (Petrov and Ma 2003) ocean tide loading displacement (OTLD) estimates. The latter study demonstrated average upper estimates for the accuracy of harmonic site position variations of $\sim 0.5 \mathrm{~mm}$ and $\sim 1.7 \mathrm{~mm}$ in the horizontal and radial coordinate components respectively.

GPS has been used by Schenewerk (2001) to measure vertical OTLD displacements at 8 diurnal and semi-diurnal frequencies. A more recent GPS-based study of OTLD (King et al. 2005) demonstrated $\sim 0.2 \mathrm{~mm}$ to $2 \mathrm{~mm}$ agreement between GPS and models in each of three coordinate components.

Superconducting gravimeters, since they were first developed in the late 1980s and 1990s, have been used to making increasingly accurate geodetic measurements, as overviewed by Crossley (2004). Baker and Bos (2003) have demonstrated that such gravity measurements were of sufficient accuracy to distinguish between different ocean tide models.

In this paper, we choose to compare GPS and VLBI measurements of OTLD since they are directly comparable (geometric) techniques. Whereas the permanent GPS network now includes several thousand sites globally, VLBI measurements, where they are made, are generally regarded as being the more accurate. However, systematic errors present in the VLBI estimates may differ from those biases affecting the GPS processing strategy, for example, multipath, satellite orbit mis-modelling, etc. The comparison of the GPS and VLBI techniques is therefore of interest, since this allows the identification and characterisation of technique-specific systematic errors. Furthermore, if GPS is capable of making measurements of harmonic ground motions with accuracies comparable to those achieved using VLBI, it opens the way for the large GPS global network to be used to test OTLD and Earth body tide models with coverage not achievable from other techniques.

To date, Petrov and Ma (2003) have provided the only systematic comparison of VLBI and GPS estimates of OTLD, comparing their own VLBI estimates of OTLD with the GPSbased values of Schenewerk et al. (2001). Using results from 28 co-located sites, Petrov and Ma (2003) found that their VLBI estimates of OTLD gave closer agreement with the model estimates than did the GPS measurements. However, King et al. (2005) showed that, around Antarctica, the earlier GPS estimates of Schenewerk et al. (2001) were not in good agreement with their own GPS estimates.

The purpose of this study, therefore, is to compare up-to-date GPS and VLBI (Petrov and Ma 2003) measurements of OTLD at co-located sites against independent estimates from modern global ocean tide models, convolved through Green's functions (Farrell 1972). 


\section{GPS estimation of OTLD}

\subsection{Site selection}

We identified permanent GPS stations that are co-located with the VLBI sites used by Petrov and $\mathrm{Ma}$ (2003) and that have the long time-series required for our approach. Typically, the GPS and VLBI sites are separated by a few metres; we permitted a maximum separation of $10 \mathrm{~km}$ in our analysis. Allinson et al.(2004) showed that in using > 1000 days of data, estimates of OTLD at the principal semi-diurnal and diurnal tidal frequencies were generally well resolved.

The 40 VLBI stations used by Petrov and Ma (2003) correspond to 34 geographically separate locations (Fig. 1, triangles). At locations with more than one VLBI antenna, and hence two or more OTLD solutions, the set of solutions with the smaller formal errors was chosen. At VLBI sites with more than one co-located GPS receiver, the longer GPS time series was used. At five of the 34 VLBI locations (KP-VLBA, LA-VLBA, OV-VLBA, NRAO20, HN-VLBA) we were unable to obtain GPS data from receivers located within 10 $\mathrm{km}$. At a further four locations, the available GPS data series was of insufficient length or quality to provide reliable OTLD estimates (BREW, HATC, KSMV, RIC1). The remaining 25 co-located locations are indicated in Fig.1 (solid triangles); broad global geographical coverage is achieved in both latitude and longitude.

Daily GPS data for these stations were collated from the International GNSS Service (IGS) (Beutler et al. 1999) sites for the period 1994.0 to 2005.0 where available. Typically, after outlier removal, there are $>3000$ days of good data for each station (Table 1).

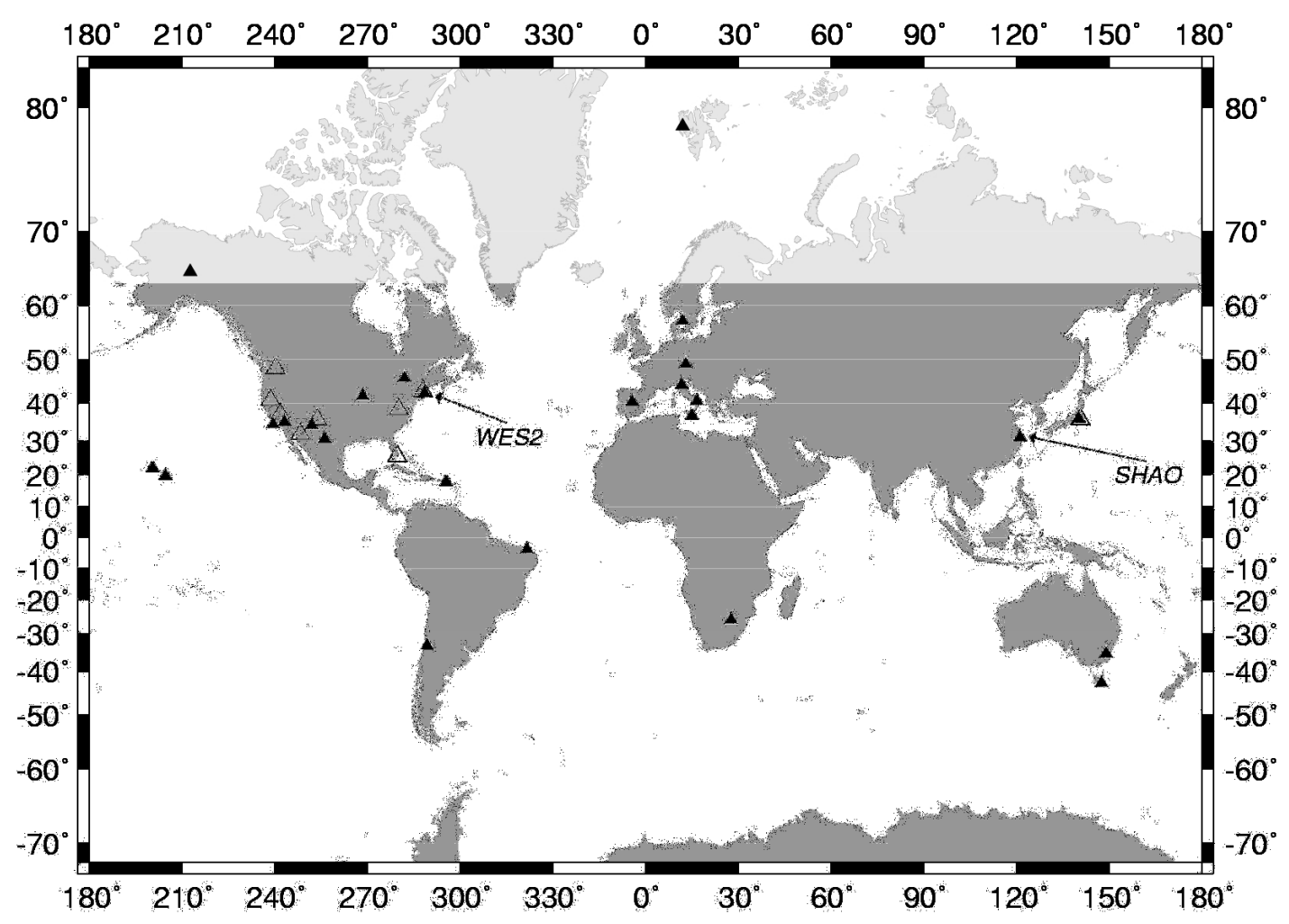


Fig. 1 The VLBI stations used by Petrov and Ma (2003) (all triangles), and the 25 co-located GPS / VLBI stations used in this study (solid triangles). Locations of sites SHAO and WES2, referred to in section 3.3, are indicated (Mercator Projection).

\begin{tabular}{|c|r|r|r|r|c|}
\hline GPS Station & Latitude & Longitude & Start Year & End year & $\begin{array}{c}\text { Data days } \\
\text { (After outlier removal) }\end{array}$ \\
\hline ALGO & 45.96 & -78.07 & 1994 & 2004 & 3944 \\
CRO1 & 17.76 & -64.58 & 1994 & 2004 & 3091 \\
FAIR & 64.98 & -147.50 & 1994 & 2004 & 3365 \\
FORT & -3.88 & -38.43 & 1994 & 2004 & 3378 \\
GOL2 & 35.42 & -116.89 & 1996 & 2004 & 3066 \\
HOB2 & -42.80 & 147.44 & 1994 & 2004 & 3184 \\
HRAO & -25.89 & 27.69 & 1996 & 2003 & 2173 \\
KOKB & 22.13 & -159.67 & 1995 & 2004 & 3223 \\
MADR & 40.43 & -4.25 & 1996 & 2004 & 2684 \\
MATE & 40.65 & 16.70 & 1994 & 2004 & 3369 \\
MDO1 & 30.68 & -104.02 & 1994 & 2004 & 3728 \\
MEDI & 44.52 & 11.65 & 1996 & 2004 & 3102 \\
MKEA & 19.80 & -155.46 & 1996 & 2004 & 2579 \\
NLIB & 41.77 & -91.58 & 1994 & 2004 & 3737 \\
NOT1 & 36.88 & 14.99 & 2000 & 2003 & 1199 \\
NYAL & 78.93 & 11.87 & 1996 & 2004 & 2653 \\
ONSA & 57.40 & 11.93 & 1994 & 2003 & 3352 \\
PIE1 & 34.30 & -108.12 & 1994 & 2004 & 3747 \\
SANT & -33.15 & -70.67 & 1994 & 2004 & 3653 \\
SHAO & 31.10 & 121.20 & 1996 & 2004 & 2352 \\
TIDB & -35.40 & 148.98 & 1996 & 2004 & 2638 \\
TSKB & 36.11 & 140.09 & 1994 & 2004 & 3762 \\
VNDP & 34.56 & -120.62 & 1994 & 2004 & 3670 \\
WES2 & 42.61 & -71.49 & 1994 & 2004 & 3652 \\
WTZR & 49.14 & 12.88 & 1996 & 2004 & 3237 \\
\hline
\end{tabular}

Table 1 GPS data used in study 


\subsection{Estimation strategy}

The GPS estimation strategy is broadly similar to that employed by Allinson et al. (2004) and King et al. (2005). The GPS data were processed on a site-by-site and day-by-day basis using the latest version (version 4) of the GIPSY / OASIS analysis software (Webb and Zumberge 1995) in its Precise Point Positioning (PPP) configuration (Zumberge et al. 1997). This version of the software (and our analysis) implements solid Earth tide models according to the IERS Conventions 2003 (McCarthy and Petit 2004).

The remaining processing parameters are identical to those used by King et al. (2005): an elevation cut-off angle of $7^{\circ}$, a sampling interval of $300 \mathrm{~s}$, and estimation of tropospheric zenith delays and gradients as random walk parameters with process noise values of $10.2 \mathrm{~mm} / \sqrt{\mathrm{hr}}$ and $0.3 \mathrm{~mm} / \sqrt{\mathrm{hr}}$ respectively. The fiducial-free precise orbits and clocks provided by the Jet Propulsion Laboratory (JPL) were employed.

Within each 24 hour PPP analysis, 51 constant parameters (three coordinate parameters, plus an additional 48 OTLD parameters) were estimated once, along with a corresponding $51 \times 51$ variance-covariance matrix. The 48 OTLD parameters represent harmonic motions (amplitudes of sine and cosine components), in each of the local co-ordinate directions (east, north, radial) at each of eight principal diurnal and semi-diurnal tidal frequencies (K1, O1, P1, $\mathrm{Q} 1$ and M2, S2, N2, K2). The sine and cosine components were loosely constrained to values of $0.000 \mathrm{~m}$ with a priori standard deviations of 0.02 and $0.2 \mathrm{~m}$ for the horizontal and radial constituents respectively to avoid numerical instabilities.

Within each daily solution, the OTLD parameters are highly correlated with each other due to the close banding of tidal frequencies at the diurnal and semi-diurnal periods. By combining the $\sim 3000$ daily solutions and their full co-variance matrices in a Kalman filter, the Rayleigh Criterion for the separation of two spectral lines (Godin 1972) is vastly exceeded and the OTLD parameters are thus de-correlated, effectively producing a weighted mean of the daily constituent estimates. Outliers were rejected using a daily estimate of unit variance.

The K2 constituent has been shown to be the slowest constituent to converge (Allinson et al. 2004), and, along with the K1 constituent, typically gives the poorest fit with both VLBI and model estimates. There is a time-variable behaviour to K2 (King 2005), possibly due to orbital effects, the period of the K2 constituent being equal to the GPS satellite orbit repeat period. To mitigate this behaviour impacting on other components through the covariance matrix, a nominal $15 \mathrm{~mm}^{2}$ of process noise was added to each of the estimated $\mathrm{K} 2$ parameters, effectively down-weighting the K2 constituent in the filter combination (King 2005). Each daily covariance matrix was scaled by the initial daily unit variance in a final iteration of the filter, producing a final unit variance close to unity.

Since the eight diurnal and semi-diurnal constituents we estimate are a small subset of tidal constituents, we add nodal corrections to each constituent to account for the modulating effects of the lunar node (McCarthy 1996; Scherneck 1999; Tamura 1987). For our long time series of $\sim 11$ years, there is a trade-off between applying the nodal corrections too early in the filter combination, when the constituents have not reliably separated for nodal corrections to be applied to the appropriate constituents, and too late, by which time which the value of the nodal corrections will have undergone a large proportion of their 18.6-year variational cycle.

To compromise, the daily OTLD estimates of the parameters were combined in the Kalman filter into yearly batch solutions, to which average nodal corrections for the year were applied, before a further iteration of the Kalman filter combined the corrected yearly solutions to give the final parameter estimate. The effect of adding nodal corrections is a small variation in OTLD parameters, of order $0.25 \mathrm{~mm}$. The amplitudes of sine and cosine components were 
finally converted to an amplitude and Greenwich phase lag per constituent, with the convention of phase lags positive.

\subsection{Ambiguity resolution}

Previous estimates of OTLD using GIPSY (Allinson et al. 2004; King et al. 2005) have not fixed carrier-phase ambiguities to integer values due to software limitations. Ambiguity fixing may, however, be important in obtaining the most reliable OTLD estimates since radial and horizontal periodic signals have previously been shown to interact via the ambiguity terms in sub-daily position estimates (King et al. 2003). To overcome this, we used a modified version of GIPSY which allowed harmonic parameters to be estimated from the ambiguity-fixed daily solutions.

Ambiguity fixing was performed in regional networks of three stations per network (Table 2). For stations in more than one network, the solution from the network with the highest ambiguity fixing rate was used. Four of the sites were too remote to attempt ambiguity fixing (HRAO, NYAL) or caused problems at the ambiguity fixing stage (HOB2, NOT1). On average, ambiguities were successfully fixed on $\sim 90 \%$ of days. The relatively low mean daily ambiguity fixing rates of between $31 \%$ and $50 \%$ (Table 2) are unsurprising, given the scale of some of the networks and the relatively long baselines between sites, particularly in the cases of networks 2, 4 and 5.

\begin{tabular}{|c|ccc|c|}
\hline Network & \multicolumn{3}{|c|}{ Stations } & \% fixed \\
\hline 1 & MDO1 & PIE1 & VNDP & 45.3 \\
2 & FAIR & GOL2 & PIE1 & 38.5 \\
3 & KOKB & TIDB & TSKB & 41.4 \\
4 & KOKB & SHAO & TSKB & 38.1 \\
5 & CRO1 & FORT & SANT & 31.1 \\
6 & ALGO & NLIB & WES2 & 40.6 \\
7 & MADR & ONSA & WTZR & 49.8 \\
8 & MATE & MEDI & WTZR & 46.8 \\
9 & KOKB & MKEA & VNDP & 51.2 \\
\hline
\end{tabular}

Table 2 Ambiguity Resolution Networks and mean daily ambiguity fixing rate.

In fixing ambiguities, it was confirmed that no significant inter-site correlations between OTLD parameters were introduced (typical correlation coefficient 0.0005 ) and hence the siteby-site combination of the daily solutions in the Kalman filter remained appropriate.

The OTLD parameter estimates from the ambiguity-fixed daily solutions were therefore combined as described for the ambiguity floating solutions. For stations and days where no ambiguity fixed solution was available, the OTLD parameters from the ambiguity floating solution were included in the combination filter. Overall, ambiguity resolution made only a small difference to the final, combined, OTLD estimates and their covariances (see section $3.2)$. 


\section{Comparison of GPS, VLBI and model OTLD}

\subsection{VLBI estimates of OTLD}

The VLBI measurements of OTLD used in this study are those derived by Petrov and Ma (2003); readers are referred to this paper for a full description of the VLBI estimation strategy. In brief, radial and horizontal positional variations of 40 VLBI stations (indicated by triangles, Fig. 1) were estimated at 32 tidal frequencies using $\sim 3 \times 10^{6}$ measurements of group delay over a 22 year period between 1980 and 2002. That study generally followed the IERS Conventions 1996 (McCarthy 1996), with the exception of the modelling of the solid Earth tides; these were expressed in the frequency domain rather than the time domain.

Petrov and Ma (2003) found VLBI estimates of OTLD to be generally in good agreement with the predictions from a number of ocean tide models, particularly at the M2 frequency. Of the eight principal diurnal and semi-diurnal constituents, discrepancies between VLBI and modelled estimates of OTLD were greatest for the S2, K1 and K2 constituents; at these frequencies the discrepancy exceeded both the formal errors of the VLBI estimates and the estimated uncertainties in the modelled values.

\subsection{Global fit of GPS and VLBI OTLD to modelled values}

Five modern global numerical ocean tide models were used in this comparison: CSR4.0 (Eanes and Bettadpur 1995), FES2004 (Lefevre et al. 2002), GOT00.2 (Ray and Ponte 2003), NAO99b (Matsumoto et al. 2000) and TPXO6.2 (Egbert and Erofeeva 2002). For each of the 25 sites, three-dimensional modelled OTLD were computed using each of these tide models by convolution with a Green's function based upon the Gutenberg-Bullen Earth model (Farrell 1972). The computation was achieved using the web based 'Ocean Tide Loading Provider' facility provided by Scherneck and Bos [http://www.oso.chalmers.se/ loading/, accessed Dec 6, 2005]. The modelled OTLD were not corrected for the (sub-millimetre) motion of the centre of mass of the solid Earth due to the ocean tides

The modelled estimates of OTLD were mainly in very close agreement with each other, and for much of the discussion we consider only the NAO99b and TPXO6.2 models. In the following discussion we have used the term 'vertical' to describe measurements that are strictly radial.

For the purposes of this study, we have assumed the model-derived estimates to be errorfree. The relative accuracies of the GPS and VLBI measurements of OTLD were assessed by comparing the fit of each to the modelled displacements. Twenty-four root mean square (RMS) misfits were calculated across the 25 sites $(n)$, for each of the eight tidal constituents (i) in each of the three component directions $(j)$ (e.g. Eq.1).

$$
\text { GPS / model rms misfit } \mathrm{i}_{\mathrm{j}, \mathrm{j}}=\left(\frac{1}{25} \sum_{n=1}^{25}\left(\operatorname{GPS}_{\mathrm{i}, \mathrm{j}, \mathrm{n}}-\operatorname{model}_{\mathrm{i}, \mathrm{j}, \mathrm{n}}\right)^{2}\right)^{1 / 2}
$$

For each of the five ocean tide models, the 24 RMS misfits were calculated for each of three comparisons: (i) GPS (ambiguity free) / model, (ii) GPS (ambiguity fixed) / model, and (iii) VLBI / model. The RMS misfits to the measurements from the NAO99b model, along with the propagated 1 sigma error bars, are shown in Fig. 2. 

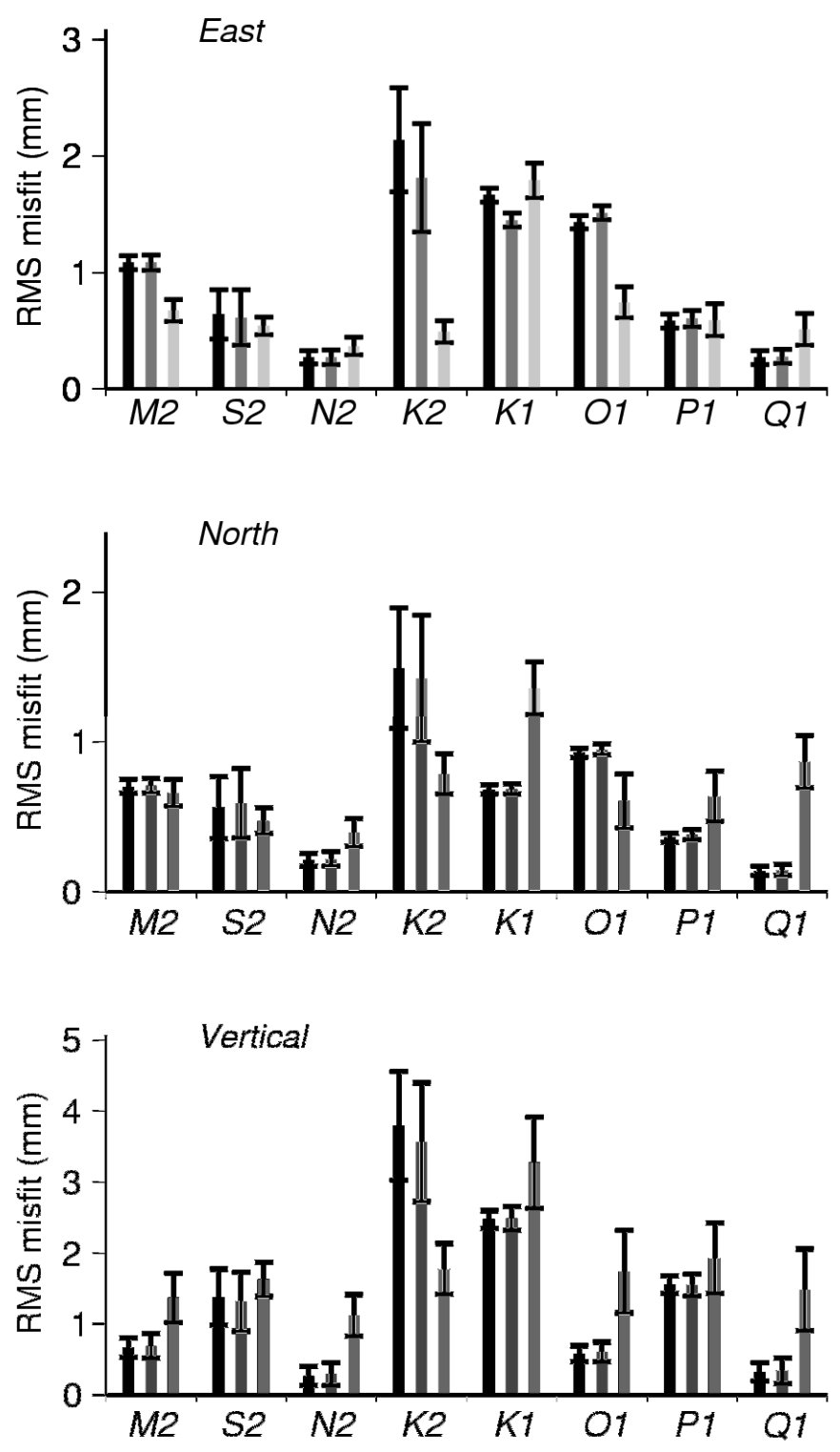

Fig. 2 Misfits to NAO99b model for eight diurnal and semi-diurnal constituents. Misfits are (i) black: GPS (ambiguity floating) / model (ii) dark grey: GPS (ambiguity fixed) / model (iii) Light grey: GPS / VLBI. One sigma formal error bars are shown.

Comparing the ambiguity-free and the ambiguity-fixed misfits to the model, it is apparent that fixing $30-50 \%$ of ambiguities to integer values has resulted in only marginal changes to the GPS / model fit. The most notable improvement in the GPS / model agreement is in the K2 constituent in all three component directions. For the K1 constituent, the GPS / model fit is slightly improved in the east, with negligible change in the other directions. There is also a marginal improvement at S2 in the vertical direction. For other constituents, the effect of ambiguity fixing is extremely small.

Comparing the GPS (fixed) / model and VLBI / model misfits in the vertical direction, the GPS / model misfit can be seen to be smaller than the VLBI / model misfit for seven of the 
eight constituents, the exception being K2. For the N2, P1 and Q1 constituents in all three component directions, the GPS / model misfit is smaller or comparable to the VLBI / model misfit. For the M2, S2 and O1 constituents in the north and east components, the GPS / model misfit is larger than the VLBI / model misfit. At K1, although comparatively large, the GPS / model misfit is smaller than the VLBI / model misfit in all three components. At K2, the GPS / model misfit is larger in all three component directions.

Sources of biases in the GPS measurement of harmonic displacements at K1 and K2 frequencies have been discussed by King (2005) and King et al. (2005). The biases at these frequencies are considered to result mainly from the orbital period of the GPS satellites corresponding to the $\mathrm{K} 2$ period, and the satellite constellation geometry repeat period corresponding to the K1 period, as discussed by Schenewerk et al. (2001). The VLBI-derived measurements are in poorest agreement when resolving displacements at the K1 period. This is likely to be due to the K1 period corresponding to the period of rotation of the Earth, and the associated trade-off between estimation of K1 harmonic parameters and UT1.

In addition to noting the relatively large K1 and K2 GPS / model misfits, we also observe that, particularly for the vertical, the GPS / model misfits are smaller for the lunar constituents, namely M2, N2, Q1 and O1, than for the solar constituents, S2, P1, K1 and K2; this is particularly evident in the vertical component. Again, we suggest that this effect is due to GPS systematic errors biasing the constituents at solar frequencies.

The corresponding TPXO6.2 misfits are shown in Fig. 3. There is little apparent difference in the pattern of misfits using either the NAO99b or TPXO6.2 models, the notable exception being the N2 constituent in the vertical direction. At this frequency, the GPS / NAO99b misfit is notably smaller than the GPS / TPXO6.2 misfit. Similarly to the NAO99b model, the GPS / model and VLBI / model misfits are largest at the K1 and K2 frequencies. The three remaining models show near identical patterns to NAO99b at all constituents, including $\mathrm{N} 2$. 

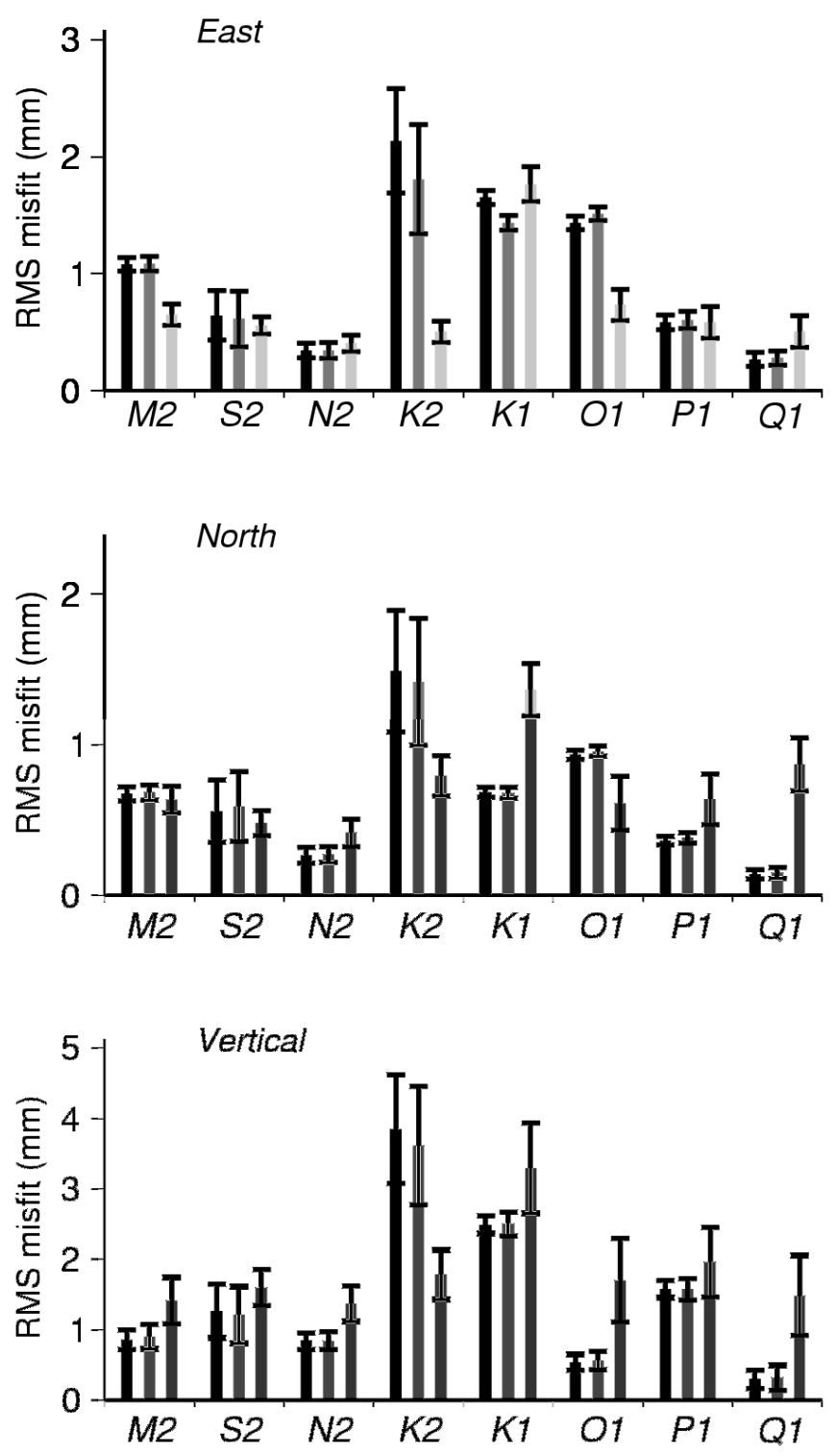

Fig. 3 Same as for Fig. 2 but using the TPXO6.2 model estimates. Note the discrepancy between TPXO6.2 and NAO99b (Fig. 2) for the GPS / model misfit at the N2 constituent.

To assess the differences in scatter between the GPS (ambiguity fixed) and VLBI measurements, a weighted chi-square statistic was calculated for each of the VLBI- and GPSderived sets of OTLD parameters, using each of the five ocean models (Eq.2).

$$
\chi^{2}=\sum_{\mathrm{i}=1}^{8(6)} \sum_{\mathrm{j}=1}^{3(1)} \sum_{\mathrm{n}=1}^{25}\left(\frac{\operatorname{GPS}_{(\mathrm{i}, \mathrm{j}, \mathrm{n})}-\operatorname{model}_{(\mathrm{i}, \mathrm{j}, \mathrm{n})}}{\sigma_{\mathrm{GPS}(\mathrm{i}, \mathrm{j}, \mathrm{n})}}\right)^{2}
$$

The statistic was calculated using the following groupings of parameters: (i) Threedimensional (complete set of 48 OTLD parameters per site), (ii) Vertical component only (16 OTLD parameters per site), (iii) Three-dimensional, excluding K1 and K2 constituents (36 
OTLD parameters per site) and (iv) Vertical component only, excluding K1 and K2 constituents (12 OTLD parameters per site). Table 3 summarizes the chi-square statistic, per degree of freedom.

\begin{tabular}{|c|c|c|c|c|c|c|c|c|c|c|c|c|}
\hline \multirow[b]{3}{*}{ Mode } & \multicolumn{6}{|c|}{ Complete set of 8 constituents } & \multicolumn{6}{|c|}{ Excluding $\mathrm{K} 1$ and $\mathrm{K} 2$ constituents } \\
\hline & \multicolumn{3}{|c|}{ Three dimensional } & \multicolumn{3}{|c|}{ Vertical } & \multicolumn{3}{|c|}{ Three dimensional } & \multicolumn{3}{|c|}{ Vertical } \\
\hline & $\begin{array}{l}\text { GPS } \\
\text { (free) }\end{array}$ & $\begin{array}{l}\text { GPS } \\
\text { (fixed) }\end{array}$ & VLBI & $\begin{array}{l}\text { GPS } \\
\text { (free) }\end{array}$ & $\begin{array}{l}\text { GPS } \\
\text { (fixed) }\end{array}$ & VLBI & $\begin{array}{l}\text { GPS } \\
\text { (free) }\end{array}$ & $\begin{array}{c}\text { GPS } \\
\text { (fixed) }\end{array}$ & VLBI & $\begin{array}{l}\text { GPS } \\
\text { (free) }\end{array}$ & $\begin{array}{c}\text { GPS } \\
\text { (fixed) }\end{array}$ & VLBI \\
\hline TPX06.2 & 4.27 & 3.63 & 2.03 & 1.22 & 0.66 & 0.64 & 3.50 & 3.13 & 1.81 & 1.18 & 0.90 & 1.63 \\
\hline NA099b & 4.23 & 3.60 & 1.90 & 1.06 & 0.53 & 0.42 & 3.43 & 3.06 & 1.66 & 0.98 & 0.72 & 1.33 \\
\hline FES2004 & 4.27 & 3.64 & 1.83 & 1.13 & 0.59 & 0.35 & 3.46 & 3.08 & 1.58 & 1.01 & 0.75 & 1.27 \\
\hline CSR4.0 & 4.40 & 3.72 & 1.96 & 1.56 & 0.89 & 0.66 & 3.65 & 3.22 & 1.73 & 1.55 & 1.13 & 1.65 \\
\hline GOT00.2 & 4.24 & 3.61 & 1.88 & 1.16 & 0.61 & 0.47 & 3.45 & 3.07 & 1.63 & 1.06 & 0.78 & 1.40 \\
\hline
\end{tabular}

Table 3 Chi-square per degree-of-freedom for GPS (ambiguity free), GPS (ambiguity fixed) and VLBI estimates of OTLD to modelled values.

There are apparent differences in the goodness of fit between the GPS and VLBI results, with the VLBI results appearing to give the better overall fit to the models in three dimensions, but the GPS results giving a comparable or better fit in the vertical direction, notably so if we exclude the noisy $\mathrm{K} 1$ and $\mathrm{K} 2$ measurements.

Paired t-tests (e.g. Field 2003) were carried out on the GPS and VLBI series of standardized residuals, $\mathrm{r}_{\mathrm{i}, \mathrm{j}, \mathrm{n}}$ (Eq.3), to assess any statistical differences between GPS and VLBI.

$$
r_{i, j, n}=\frac{\left(\operatorname{GPS}_{i, j, n}-\operatorname{model}_{i, j, n}\right)}{\sigma_{g p s i, j, n}}
$$

These tests provided no evidence of statistically significant differences, at the $95 \%$ confidence level, between the GPS and VLBI fits to any of the five sets of modelled OTLD, or between any of the four groupings of parameters.

\subsection{Spatial Variations}

Spatial variation in the amplitudes and phases of the GPS / model misfits was investigated in terms of their latitudinal and longitudinal variability, using the misfits computed from the TPXO6.2 model. From our 25 sites analysed, there was no apparent spatial pattern in amplitude or phase of the GPS / model misfits in either latitude or longitude.

Continuous gravity observations (Baker and Bos 2003; Llubes and Mazzega 1997), using a different spatial sampling to this study, have previously established that there is not a single global ocean tide model that fits observations equally well globally and that models perform differently in different regions. Whilst we have shown that GPS is not capable of distinguishing between the five models in an overall global comparison of OTLD, there are certain sites and constituents where GPS can separate the models at a 95\% confidence level. As an illustration, Figures 4 and 5 show sample phasor plots of GPS, VLBI and model 
estimates of OTLD for the M2 and O1 constituents at sites SHAO and WES2 (Fig. 1) respectively.
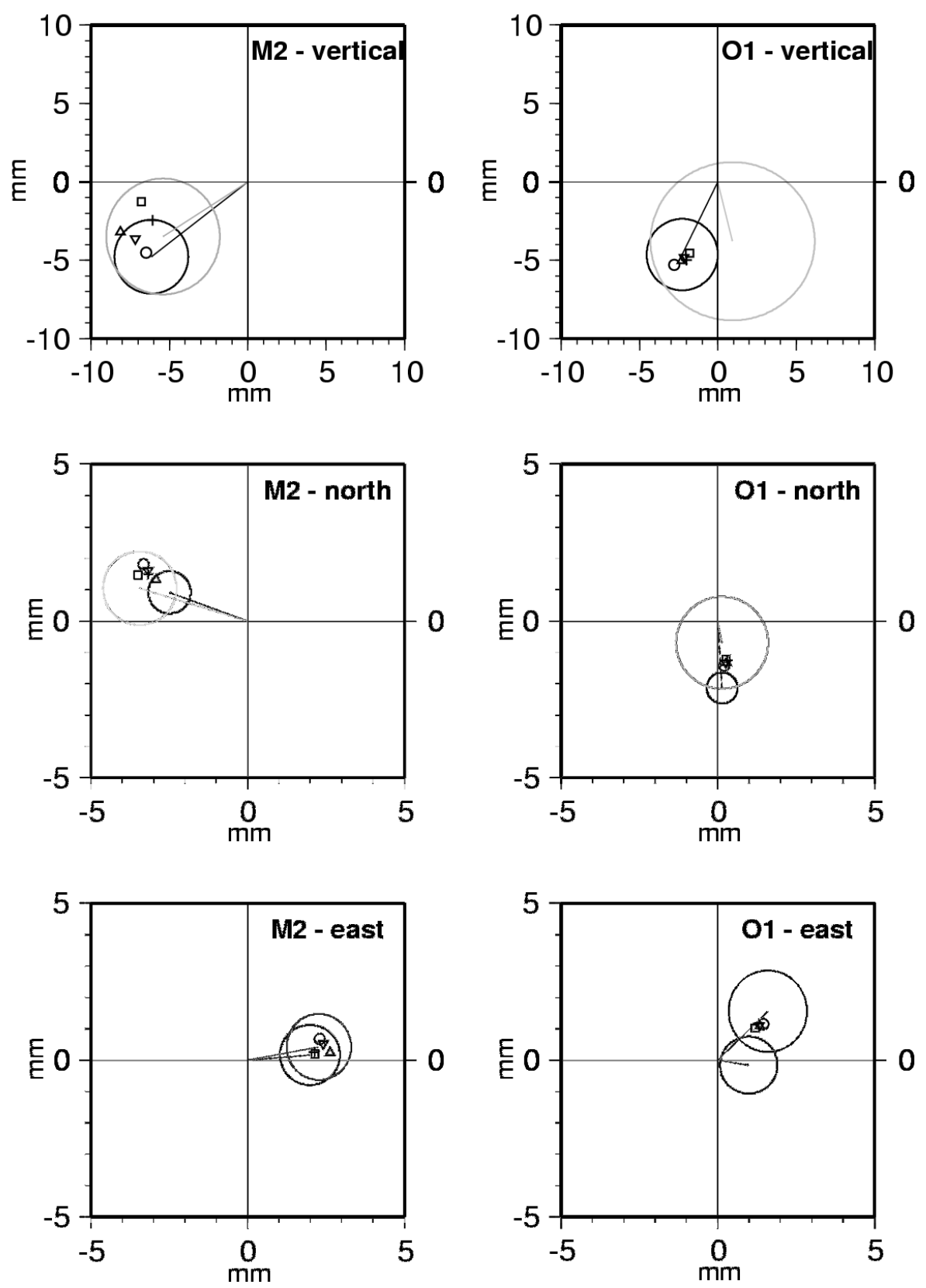

Fig. 4 Phasor plots of GPS (black line), VLBI (grey line) and modelled (symbols) estimates of OTLD for M2 and $\mathrm{O} 1$ constituents, at site SHAO. 95\% error ellipses are indicated for GPS and VLBI estimates. (TPXO6.2: triangle, NAO99: circle, FES2004: cross, CSR4.0: square, GOT00.2: inverted triangle) 

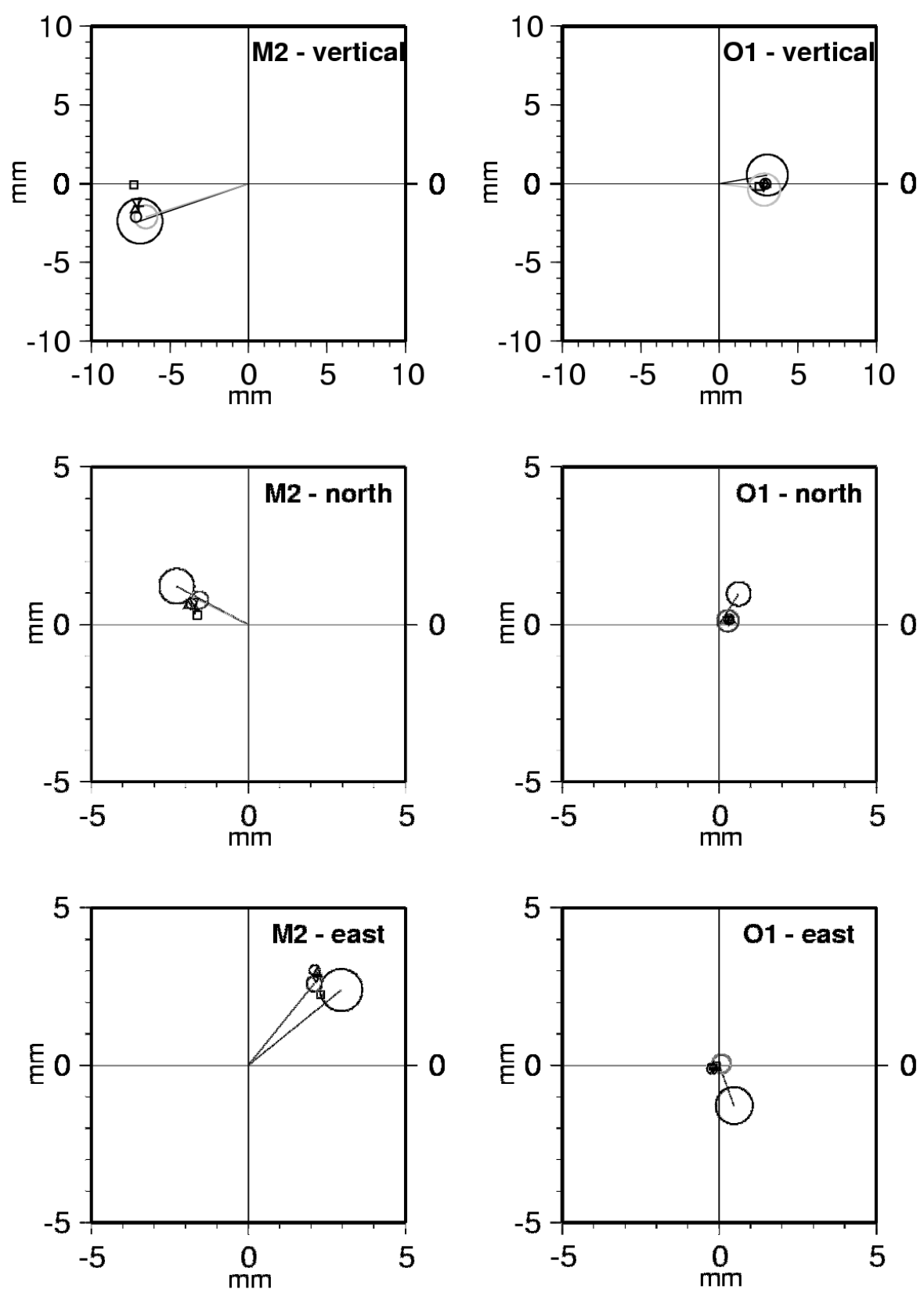

Fig. 5 Same as Fig. 5 for site WES2.

At SHAO, GPS measurements of the M2 constituent in the vertical component can (unlike VLBI) distinguish between the different modelled OTLD with 95\% confidence; of the five models, only the FES2004 and GOT00.2 estimates agree with the GPS estimate. In the north component, only the TPXO6.2 model agrees with GPS, whilst in the east component GPS, VLBI and all five models are in agreement. The plots for the O1 constituent at SHAO clearly illustrate differences in the capability of GPS measurements of OTLD in vertical and horizontal component directions. The vertical estimate agrees with all models (and disagrees with VLBI) whilst the north, and more particularly the east component give poor agreement 
with VLBI and all five models. This pattern of agreement for the $\mathrm{O} 1$ constituent at SHAO is typical of all sites, as can be seen from the $\mathrm{O} 1$ global misfit in Figures 2 and 3.

At WES2, GPS measurement of the M2 constituent in the vertical direction disagrees with only the NAO99b model at the $95 \%$ confidence level. In the north component, VLBI agrees with some but not all models at 95\% confidence, whilst GPS does not agree with any model. In the east component, GPS and VLBI agree with different models at the 95\% confidence level - the GPS estimate agrees with only the NAO99b estimate. For the O1 constituent in the vertical direction, as for SHAO, the GPS agrees well with all five models and the VLBI estimates. GPS is considerably less accurate than VLBI at estimating the small horizontal O1 signals, significantly over-estimating the amplitude.

\section{Conclusions}

We have demonstrated that GPS and VLBI data are similarly useful in determining OTLD estimates at diurnal and semi-diurnal frequencies, in both vertical and horizontal directions, for a globally distributed selection of stations.

By comparison of GPS and VLBI measurements of OTLD with various ocean tide model predictions, we conclude that GPS is capable of estimating the important vertical OTLD displacements with accuracy equal to or greater than that achieved from VLBI, for all constituents except the K2 frequency. At the K1 frequency, the GPS and VLBI estimates are both of relatively low accuracy.

For a global network, the fixing of ambiguities to integer values has marginal benefit on the accuracy of OTLD measurement, the most notable improvement being at the K2 frequency.

At certain individual sites and in certain components / constituents, we have shown that GPS is capable of distinguishing between the modelled OTLD at a 95\% confidence level. We have shown that overall, however, the fit of modelled estimates of OTLD to both GPS and VLBI measurements is similar for the five ocean tide models tested, and therefore we can not distinguish between the models at global scale. We have also demonstrated that there is no statistical difference between the GPS / model fits and the VLBI / model fits. Therefore we conclude that GPS is capable of measuring OTLD with comparable accuracy to VLBI, and within the accuracy of present numerical OTLD models.

\section{Acknowledgements}

We would like to thank Leonid Petrov of NASA Goddard Space Flight Centre for making his VLBI estimates of harmonic displacements freely available via the Internet, and also the International GNNS Service community for supplying daily GPS data. The NASA Jet Propulsion Laboratory (JPL) contributed significantly by providing modified GIPSY routines, and their orbit, clock and Earth orientation products. The UK Engineering and Physical Science Research Council provided financial support to Ian Thomas through a Doctoral Training Account award to Newcastle University. Matt King was supported by a UK Natural Environment Research

Council postdoctoral fellowship. 


\section{References}

Allinson CR, Clarke PJ, Edwards SJ, King MA, Baker TF, Cruddace PR (2004) Stability of direct GPS estimates of ocean tide loading. Geophys. Res. Lett. 31(15): doi:10.1029/2004GL020588, 2004.

Baker TF, Bos MS (2003) Validating Earth and ocean tide models using tidal gravity measurements. Geophysical Journal International 152(2): 468-485.

Beutler G, Rothacher M, Schaer S, Springer TA, Kouba J, Neilan RE (1999) The International GPS Service (IGS): An Interdisciplinary Service in Support of Earth Sciences. Adv. Space Res. 23(4): 631-635.

Crossley D (2004) Preface to the Global Geodynamics Project. Journal of Geodynamics 38(3-5): 225-236.

Eanes RJ, Bettadpur SV (1995) The CSR 3.0 global ocean tide model. Center for Space Research, Technical Memorandum CSR-TM-96-05.

Egbert GD, Erofeeva L (2002) Efficient inverse modeling of barotropic ocean tides. Journal of Atmospheric and Oceanic Technolgy 19.

Farrell WE (1972) Deformation of Earth by Surface Loads. Reviews of Geophysics and Space Physics 10(3): 761-797.

Field A (2003) 'Discovering Statistics: using SPSS for Windows.' (SAGE Publications Ltd.)

Godin G (1972) 'The Analysis of Tides.' (Liverpool University Press)

King M (2005) Kinematic and static GPS techniques for estimating tidal displacements with application to Antarctica. Journal of Geodynamics, doi:10.1016/j.jog.2005.08.019.

King M, Coleman R, Nguyen LN (2003) Spurious periodic horizontal signals in sub-daily GPS position estimates. Journal of Geodesy 77(1-2): 15-21, doi:10.1007/s00190-00002-00308-z.

King MA, Penna NT, Clarke PJ, King EC (2005) Validation of ocean tide models around Antarctica using onshore GPS and gravity data. Journal of Geophysical Research-Solid Earth 110(B8): B08401, doi: 10.1029/2004JB003390, 2005.

Lefevre F, Yard FH, Le Provost C, Schrama EJO (2002) FES99: A global tide finite element solution assimilating tide gauge and altimetric information. Journal of Atmospheric and Oceanic Technology 19(9): 1345-1356.

Llubes M, Mazzega P (1997) The ocean tide gravimetric loading reconsidered. Geophysical Research Letters 23(12): 1481-1484.

Matsumoto K, Takanezawa T, Ooe M (2000) Ocean Tide Models Developed by Assimilating TOPEX/POSEIDON Altimeter Data into Hydrodynamical Model: A Global Model and a Regional Model around Japan. Journal of Oceanography 56567-581.

McCarthy DD, Petit G (Eds) (2004) IERS Technical Note 32. IERS Conventions (2003), International Earth Rotation and Reference Systems Service (IERS), Frankfurt am Main, Germany: Verlag des Bundesamtes für Kartographie und Geodäsie.

McCarthy DD (Ed.)(1996) IERS Technical Note 21. IERS Conventions (1996), International Earth Rotation and Reference Systems Service (IERS), Frankfurt am Main, Germany: Verlag des Bundesamtes für Kartographie und Geodäsie.

Petrov L, Ma CP (2003) Study of harmonic site position variations determined by very long baseline interferometry. Journal of Geophysical Research 108(B4): 2190, doi: 10,1029/2002JB001801, 2003. 
Ray RD, Ponte RM (2003) Barometric tides from ECMWF operational analyses. Annales Geophysicae 21(8): 1897-1910.

Schenewerk MS, Marshall J, Dillinger W (2001) Vertical ocean-loading deformations derived from a global GPS network. Journal of the Geodetic Society of Japan 47(1): 237-242.

Scherneck H-G (1999) Explanatory supplement to the section "Local site displacement due to ocean loading" of the IERS Conventions. Explanatory supplement to the IERS Conventions (1996) Chapters 6 and 7.

Sovers OJ (1994) Vertical Ocean Loading Amplitudes from VLBI Measurements. Geophysical Research Letters 21(5): 357-360, doi: 0094-8534/94/93GL-02648\$03.00.

Tamura Y (1987) A harmonic development of the tide-generating potential. Bull. Inf. Mar. Terr. 996813-6857.

Webb FH, Zumberge JF (1995) An introduction to GIPSY/OASIS-II precision software for the analysis of data from the Global Positioning System. Report D-11088Jet Propulsion Laboratory, Pasadena.

Zumberge JF, Heflin MB, Jefferson DC, Watkins MM, Webb FH (1997) Precise point positioning for the efficient and robust analysis of GPS data from large networks. Journal of Geophysical Research 102(B3): 50055017. 